\title{
Gemeinwesenarbeit im Netz
}

\section{Die QuarterNet-Datenbank sammelt Informationen zur Gemeinwesenarbeit}

\author{
Anette Becker und H. Jürgen Stuppi
}

Anette Becker arbeitet als DiplomSozialpädagogin (FH) seit 20 Jahren beim Caritasverband für Saarbrücken und Umgebung e. V. im Gemeinwesenprojekt CaritasKontaktzentrum Folsterhöhe. Sie ist zuständig für die QuarterNetDatenbank der Gemeinwesenarbeit, Rubrik »Veröffentlichungen \& Praxishilfen «. Seit 2009 ist sie zusätzlich auch Referentin bei der Landesmedienanstalt Saar. E-Mail a.becker@quarternet.de

\section{H. Jürgen Stuppi ist Diplom-} Sozialarbeiter und Geschäftsführer des Paritätischen Bildungswerkes Rheinland-Pfalz/Saarland e. V. Gemeinsam mit Dr. Armin Kuphal ist er Initiator des Projektes QuarterNet und für dessen Koordination verantwortlich. E-Mail juergen.stuppi@paritaet.org
Die Datenbank QuarterNet.de ist seit zwölf Jahren eine gute Adresse im World Wide Web, wenn es um aktuelle und grundlegende Informationen zur Gemeinwesenarbeit, zum Quartiersmanagement und zur Stadtteilentwicklung geht. Koordinator des kostenlosen Online-Angebots ist das Paritätische Bildungswerk in Rheinland-Pfalz/ Saarland.

In der QuarterNet-Datenbank werden Informationen zur Gemeinwesenarbeit aus Deutschland und den Nachbarstaaten zusammengetragen. Die Datenbank bietet damit erstmals die Möglichkeit der bundesweiten Eingabe von Informationen zur Gemeinwesenarbeit mit fachspe- zifischer Suchfunktion. Das Besondere daran: Sie wird seit ihrem Modellstart im Jahr 1999 weiterhin mit Engagement und trägerübergreifend von Expertinnen und Experten aus der Gemeinwesenarbeit gepflegt.

Die QuarterNet-Datenbank wurde speziell nach inhaltlichen Vorschlägen aus der Gemeinwesenarbeit programmiert und bietet drei unterschiedliche Themenfelder, so dass alle Informationen jeweils zielgenau eingegeben und treffsicher gefunden werden können:

- Adressen: Kontakte mit Projekten und Einrichtungen (Gemeinwesenprojekte, Stadtteilarbeit, Nachbarschafts- und Bürgerzentren), Arbeitskreisen und Netzwerken

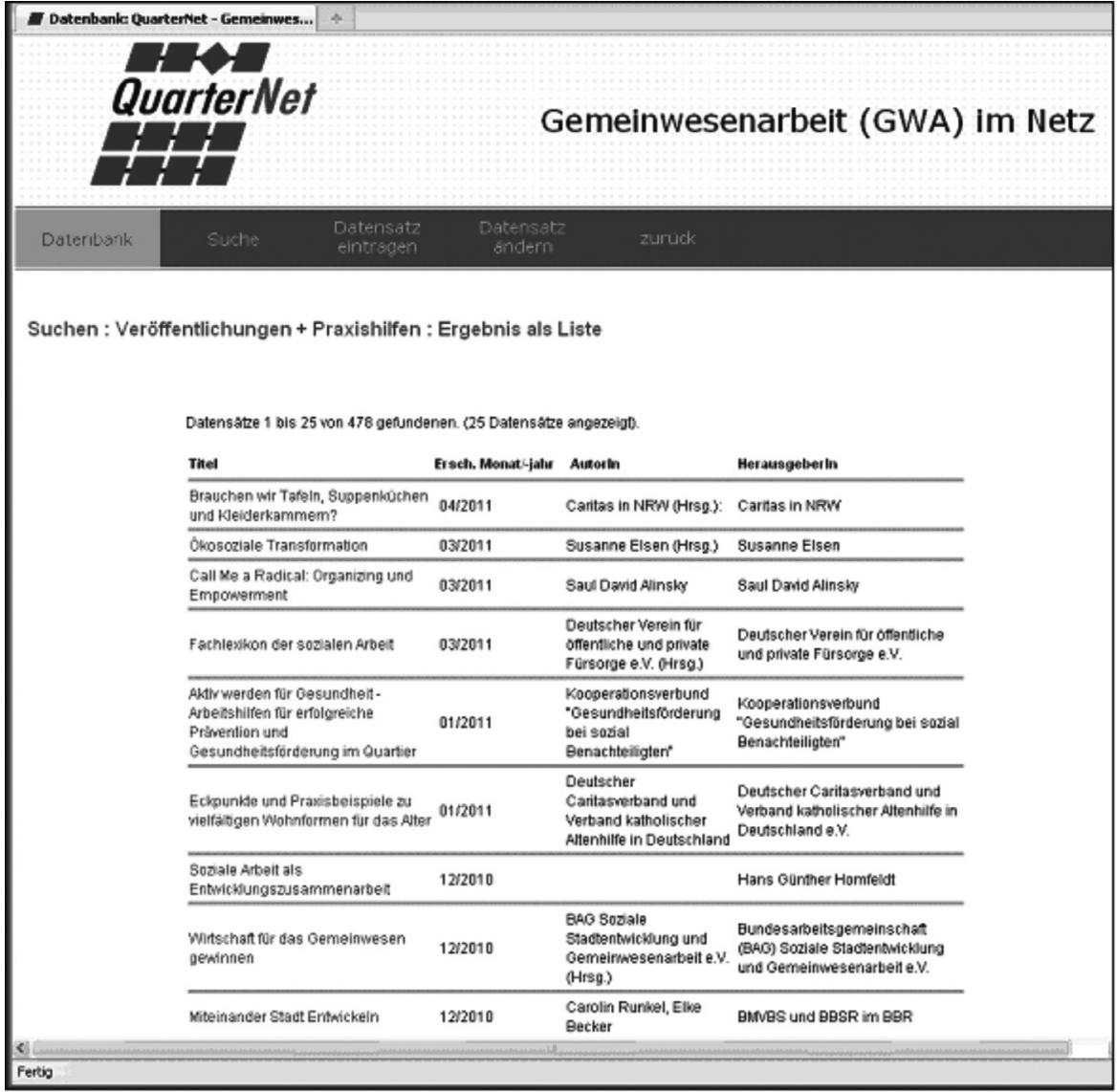

Die Suchergebnisse in der QuarterNet-Datenbank zur Gemeinwesenarbeit werden detailliert und übersichtlich dargestellt. 
- Qualifizierung: Veranstalter für Fortund Weiterbildungen

- Medien: Stadtteilzeitungen sowie Veröffentlichungen \& Praxishilfen

Insgesamt finden sich bisher mehr als 600 Beiträge in der Datenbank der Gemeinwesenarbeit. Alle praxisorientierten Einträge informieren mit inhaltlichen Beschreibungen über den Eintrag, zeigen die Kontaktdaten der Autorinnen und Autoren, der Herausgeberinnen und Herausgeber, die aktuelle Bezugsquelle oder bieten eine direkte Download-Möglichkeit.

Herzstück ist das Themenfeld »Veröffentlichungen \& Praxishilfen « mit seinen fast 500 empfehlenswerten Beiträgen aus allen Aktionsfeldern der Gemeinwesenarbeit. Hier werden Praxishandbücher, Projektvorstellungen, Konzepte und Methodenbücher gesammelt - aus der Stadtund Quartiersentwicklung, der lokalen Ökonomie und aus dem Bereich der Beteiligung und Aktivierung, Partizipation und dem Community Organizing. Bereichert wird die Datenbank um aktuelle Analysen, Studien aus dem Feld der Migration und Fachartikel beispielsweise zum Thema Armut und Kinderarmut.

\section{Finden statt suchen}

Viele Beiträge, wie die Merkblätter zu Hartz IV, sind von Praktikern aus der Gemeinwesenarbeit und Beratungsstellen erarbeitet und exklusiv von dieser Website herunterzuladen. Jahresberichte und Dokumentationen von Fachtagungen sind ebenfalls vorhanden. Ergänzt werden die
Beiträge aus der Praxis mit ausgewählter und empfehlenswerter aktueller Fachliteratur. Neu hinzugekommen sind vor kurzem das Thema Gesundheitsförderung und der Bereich »Leben und Wohnen im Alter .

Eine detaillierte Suchfunktion erschließt die Datenbank der Gemeinwesenarbeit mit unterschiedlichen Recher- diums Internet, so ist QuarterNet heute zu einem wichtigen und festen Bestandteil der fachlichen Arbeit in der Gemeinwesenarbeit geworden. Der überregional besonders interessante Teil von QuarterNet gliedert sich in die Bereiche:

- News und Termine: In dieser Rubrik informiert QuarterNet über tagesaktuelle Informationen aus der Gemein-

\section{"Mehr als 600 Beiträge informieren über Praxis und Theorie der Gemeinwesenarbeit «}

che-Möglichkeiten, so dass die Nutzer die passende und treffsicherste Option wählen können. Mit der aktuellsten Suchoption "Alle anzeigen « werden alle Einträge chronologisch nach Erscheinungsdatum sortiert. Und über das erweiterte Service-Angebot der »Detaillierten Suche « werden zielgenau und schnell alle Beiträge gefunden, in denen der eingegebene Suchbegriff vorkommt, beispielsweise Autor, Titel oder der nur noch auszuwählende Arbeitsschwerpunkt aus der Gemeinwesenarbeit (vgl. Abb.).

Vor über zehn Jahren initiierte das $\mathrm{Pa}-$ ritätische Bildungswerk RheinlandPfalz/Saarland das digitale Netzwerk der Gemeinwesenarbeit. Herrschte zu Projektbeginn bei den Akteuren noch eine gewisse Skepsis über den Nutzen und Mehrgewinn hinsichtlich des neuen Me- wesenarbeit und dem Quartiersmanagement.

- Links: In 22 Kategorien finden sich circa 150 praxisorientierte Linkadressen mit hilfreichen Informationen rund um die Gemeinwesenarbeit. Die Linkliste wird permanent gepflegt und die einzelnen Linkadressen werden erläutert.

- Dritter Bereich ist die oben beschriebene QuarterNet-Datenbank der Gemeinwesenarbeit.

Im regionalspezifischen Teil "Saarland « und »Rheinland-Pfalz « findet sich eine Übersicht der Akteure der Gemeinwesenarbeit, der Zugang zum Intranet der Gemeinwesenarbeit im Saarland sowie die digitale Stadtteilzeitung "guggemol .

Internet http://www.quarternet.de und http://www.gemeinwesenarbeit.de

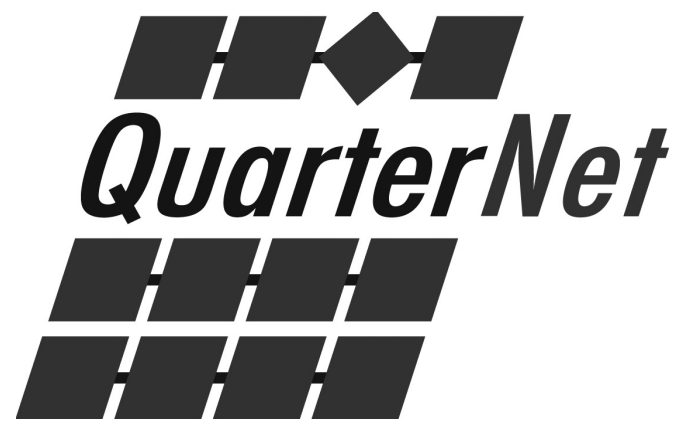

Eine Datenbank lebt von ihren Einträgen. Zum Ausbau der nutzbringenden bundesweiten Datenbank QuarterNet sind die Betreiber auf Informationen der Akteure im Arbeitsfeld der Gemeinwesenarbeit und der Quartiersentwicklung angewiesen - sowohl von Praktikerinnen und Praktikern als auch von Mitarbeitenden aus Forschung und Lehre. Die Betreiber laden deshalb alle Interessenten herzlich ein, ihr Projekt, ihr Netzwerk, ihre Stadtteilzeitung und ihre Praxishilfen in der QuarterNet-Datenbank der Gemeinwesenarbeit zu veröffentlichen. $\mathrm{Zu}$ allen Themenfeldern stehen hierzu einfache Online-Formulare zur Verfügung, mit denen unkompliziert die Einträge (»Datensatz eintragen «) zugeschickt werden können. Die einzelnen Betreuer der Rubriken (»Patinnen « und »Paten «) aus Gemeinwesenprojekten im Saarland schalten die Beiträge frei und stehen auch gerne für Fragen und für weiteren Austausch zur Verfügung.

Kontakt: E-Mail datenbank@quarternet.de 